\title{
Généralités sur les transferts turbulents dans un gradient thermique vertical
}

\author{
par M. Coantic \\ Institut de Mécanique Statistique de la Turbulence, \\ Marseille
}

\section{1 - Description physique qualitative}

Dans la première partie de cet exposé, nous allons rappeler certains schémas qualitatifs simplifiés destinés à faciliter la compréhension du mécanisme des transferts turbulents, et cles modifications qui lui sont apportéees par un gradient thermique vertical en présence d'un champ de pesanteur.

\section{1 - Situation de stabilité neułre}

Considérons un milieu fluide, dans un état d'écoulement turbulent, et dont la vitesse moyenne, $\bar{U}$, est parallèle à une direction horizontale $O x$ (voir fig. 1 a). La masse spécifique $p$, du fluide est supposée uniforme, mais la répartition moyenne d'une grandeur, $X$, transportable par le fluide est une fonction de la profondeur, $\bar{X}(z)$, que nous supposerons croissante, pour fixer les idées. Par suite du mouvement d'agitation turbulente, les particules fluides qui se succèdent en un point $P$, à la profondeur $z_{0}$, avaient auparavant séjourné à des niveaux différents, tels que $z_{1}$, où l'on peut supposer qu'elles avaient acquis en moyenne la valeur locale, $\bar{X}\left(z_{1}\right)$, de la grandeur transportable. Si $z_{1}$ est inférieur à $z_{0}$, la fluctuation verticale de vitesse associée à la particule $w^{\prime}$, est en moyenne positive, mais la fluctuation correspondante de la grandeur $X$, soit:

$$
x^{\prime}=\bar{X}\left(z_{1}\right)-\bar{X}\left(z_{0}\right)
$$

est en moyenne négative, et le produit $x^{\prime} w^{\prime}$ est le plus souvent négatif. $\mathrm{Si}$, au contraire, $z_{1}$ est supérieur à $z_{0}$, les deux fluctuations changent de signe, mais leur produit reste le plus souvent négatif. Or, le produit $x^{\prime} w^{\prime}$ représente le débit instantané de la grandeur transportable à travers un élément unitaire de surface, horizontal et centré en $P$. Sa valeur moyenne, $\overline{x^{\prime} w^{\prime}}$, correspond donc au taux moyen de transfert par la turbulence à travers cet élément, ou «flux turbulent» de $X$ suivant la direction verticale $J_{X, z}$. D'après ce qui précède, $J_{X, \approx}$ est vraisemblablement du signe opposé à celui de $\partial X / \partial z$, et d'autant plus élevé que les mouvements turbulents verticaux sont plus intenses. On peut alors poser :

$$
J_{X, z}=\overline{x^{\prime} w^{\prime}}=-K_{x, *} \frac{\partial \bar{X}}{\partial z}
$$

ce qui définit le «coefficient de diffusivité turbulente apparente de $X », K_{X, \Sigma}$, grandeur essentiellement positive et de dimensions $L-T^{-1}$. Le concept de diffusivité turbulente, initialement proposé par Boussinesq, a été largement utilisé en océanographie, mais ses limitations sont aujourd'hui bien connues (voir, par exemple, Tennekes et Lumley, 1972). On notera tout particulièrement ici que, si la diffusivité moléculaire est une propriété physique du fluide, la diffusivité turbulente est une caractéristique de l'écoulement qui dépend au premier ordre du niveau et de la structure des mouvements turbulents dans la direction considérée.

Dans la situation considérée jusqu'ici, dite de stabilité neutre ou de stratification indifférente, la masse spécifique du fluide étant supposée uniforme, ces mouvements ne sont pas influencés par la pesanteur, puisque les particules possèdent à tout instant la même densité que le fluide environnant.

\section{2 - Sifuation de faible stabilité}

Considérons à présent un milieu fluide présentant un gradient thermique vertical tel que la masse spécifique soit une fonction de la profondeur, $p(z)$, que nous supposons 

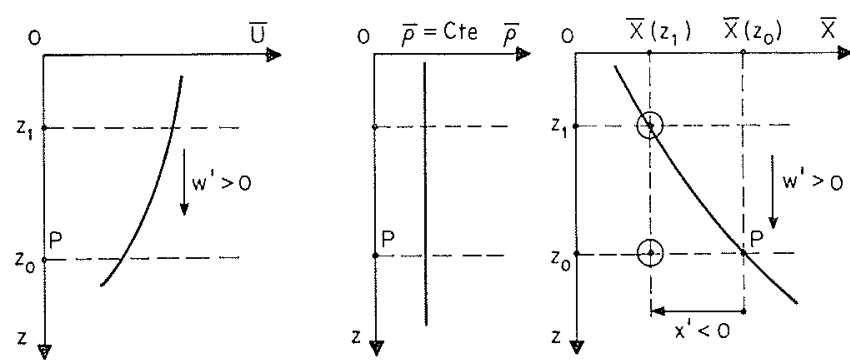

(a) Stabilité neutre, $\overline{x^{\prime} w^{\prime}}=-k_{x, z} \frac{\partial \bar{x}}{\partial z}$
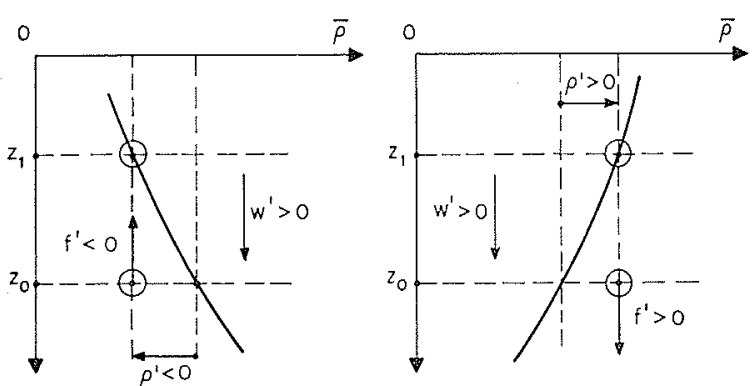

(b) Faible stabilité, $f^{\prime} w^{\prime}<0$

(c) Faible instabilité, $f^{\prime} w^{\prime}>0$

1/ Les transferts turbulents au voisinage de la stabilité neutre.

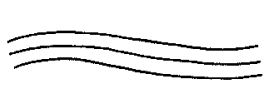

(1)

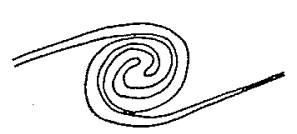

(4)

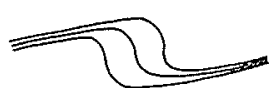

(2)

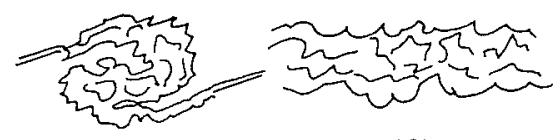

(5)

(6)
2/ Les étapes successives de la transformation d'une onde interne en une couche turbulente, par instabilité de Kelvin-Helmholtz, d'après Thorpe (1969).

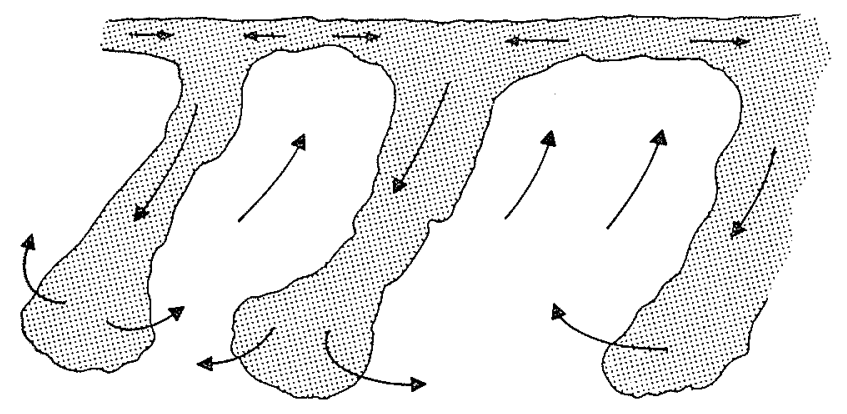

3/ Struclure schématique de l'écoulement dans une sifuation de forte instabilité; les zones en grisé correspondent aux régions du fluide où la densité est plus élevée. pour l'instant faiblement croissante (voir fig. 1 b). Les mouvements d'agitation turbulente sont alors nécessairement influencés par la pesanteur, puisqu'ils transfèrent des particules possédant en moyenne la densité correspondant à leur niveau initial, $\bar{\rho}\left(z_{1}\right)$, à des niveaux où la densité moyenne, $\bar{\rho}\left(z_{0}\right)$, est différente. La force d'Archimède s'exerçant sur un volume fuicle unitaire,

$$
j^{\prime}=g\left[\bar{p}\left(z_{1}\right)-\bar{p}\left(z_{0}\right)\right]=g p^{\prime}
$$

tend naturellement à ramener la particule au niveau où elle avait séjourné antérieurement. Le travail correspondant, $f^{\prime} w^{\prime}=g \rho^{\prime} w^{\prime}$, est nécessairement négatif et doit donc être fourni aux dépens de l'énergie cinétique du mouvement turbulent, dont l'amplitude est diminuée et la structure modifiée. On dit alors que le gradient thermique vertical est stable, puisque la répartition des densités agit à l'encontre des fluctuations turbulentes. Pour une répartition donnée de la grandeur transportable, il est clair que le flux turbulent et la diffusivité apparente qui lui correspond sont plus faibles que dans le cas précédent: la stratification stable favorise moins les transferts turbulents que la stratification indifférente.

\section{3 - Situation de forte stabilité}

Admettons maintenant que le gradient thermique vertical est suffisamment élevé pour que la masse spécifique augmente très rapidement en fonction de la profondeur. On conçoit aisément que l'obstacle opposé par les forces d'Archimède aux mouvements turbulents verticaux soit tel que ces dernicrs cessent pratiquement d'exister. Dans cette situation, dite "de forte stabilité », le caractère de l'écoulement est donc profondément modifié, et divers phénomènes vont apparaître en fonction des circonstances:

1.3.1 - Si le gradient vertical de la vitesse moyenne, $\partial \bar{U} / \partial z$, reste suffisamment faible, le mouvement d'agitation turbulente peut complètement disparaître. Il n'en faudrait pas conclure pour autant que les mouvements verticaux sont complètement exclus : c'est leur nature seulement qui change. Un milieu fluide fortement stable présente en effet une certains analogie avec un pendule pesant: il reste au repos dans sa position d'équilibre, mais oscille autour de cette position si une perturbation lui est apportée. Ainsi se développent au sein des régions de forte stratification des ondes internes de divers types (voir, par exemple, Phillips, 1966, et Turner, 1973). La fréquence propre des petites oscillations se déduit aisément des équations linéarisées qui sont alors valables pour la masse et la quantité de mouvement verticale :

$$
\frac{\partial \rho^{\prime}}{\partial t}+w^{\prime} \frac{\partial \overline{\hat{s}}}{\partial z}=0, \frac{\partial w^{\prime}}{\partial t}=+g \frac{\rho^{\prime}}{\bar{\rho}}
$$

D'où, en éliminant $\rho^{\prime}$, l'équation différentielle:

$$
\frac{\partial^{2} w^{\prime}}{\partial t^{2}}=\sim\left(\frac{g}{\bar{g}} \frac{\partial \bar{\rho}}{\partial z}\right) w^{\prime}
$$

qui admet pour solution une oscillation harmonique de fréquence: 


$$
N=\left(+\frac{g}{\bar{\rho}} \frac{\partial \bar{\rho}}{\partial z}\right)^{1 / 2}
$$

que l'on appelle la «fréquence de Brunt-Vaïsälä ».

Une autre manière d'aborder la question (qui correspond d'ailleurs souvent mieux à la réalité physique) est de dépeindre le milieu stratifié comme la superposition d'un grand nombre de couches ou «feuillets » horizontaux, dont la densité croît d'une couche à l'autre avec la profondeur. Des ondes internes peuvent alors se développer à chacune des interfaces, avec des propriétés analogues à celles des ondes de surface, et notamment la possibilité de transférer de la quantité de mouvement, mais non de la chaleur ou de la masse. Ces ondes peuvent présenter un caractère aléatoire qui les fait alors aisément confondre avec des mouvements proprement turbulents.

1.3.2 - Lorsque la fréquence caractéristique correspondant au gradient vertical de vitesse cesse d'être petite vis-à-vis de la fréquence de Brunt-Vaïsälä, les ondes internes deviennent instables. Leur amplitude croît, elles s'enroulent sur elles-mêmes et «déferlent» en donnant naissance à une série de «rouleaux» turbulents, et une couche turbulente finit par s'établir sur une certaine hauteur (voir fig. 2). Ce mécanisme est connu sous le nom d'instabilité de KelvinHelmholtz et considéré comme la source la plus fréquente de la turbulence au sein des zones stables de l'océan et de l'atmosphère (voir, par exemple, les collections IUCRM de Stockholm, 1969, et La Jolla, 1972). Pour qu'il se déclenche à partir d'oscillations de petite amplitude, il est nécessaire (cf. Turner, 1973, ch. 4) que le nombre de Richardson de gradient, défini par :

$$
\mathrm{Ri}=\left(\frac{N}{\partial \bar{U} / \partial z}\right)^{2}=+g \frac{\partial \bar{\rho} / \partial z}{\rho(\partial \bar{U} / \partial z)^{2}}
$$

devienne localement inférieur à une valeur critique de $1 / 4$.

1.3.3 - La plus grande partie du volume occupé par les océans est dans un état de stratification stable, avec des périodes de Väisälä comprises entre quelques minutes, dans les zones de fort gradient de densité comme la thermocline, et quelques heures dans les couches profondes. Les mouvements à caractère turbulent qui s'y développent sont donc très généralement confinés dans des régions quasi-horizontales, en forme de "galettes» (appelées pancakes en anglais et blini en russe), séparées par des volumes relativement libres de turbulence, mais non exempts d'ondes internes. Les mouvements turbulents à grande échelle responsables de la diffusion des contaminants scalaires, tels que chaleur, sel, composés chimiques et polluants divers, sont donc considérablement plus développés horizontalement que verticalement. Ceci explique pourquoi les coefficients de diffusivité turbulente apparente horizontale, $K_{X, v}, K_{X, y}$, sont en général au moins mille fois plus élevés que le coefficient de diffusivité verticale, $K_{X, z}$ (cf. Lacombe, 1965, p. 136).

Il faut insister par ailleurs sur le fait que la quantité de mouvement peut être transférée aussi bien par les mouvements de nature ondulatoire que par ceux présentant un caractère turbulent, alors que seuls les derniers sont capables de transporter les contaminants scalaires. Dans la situation générale décrite ci-dessus, les nombres de Prandtl et de Schmidt effectifs, correspondant aux rapports des diffusivités apparentes de ces différentes variables, tendent donc vraisemblablement vers des valeurs élevées.

\section{4 - Situation de faible instabilité}

Considérons maintenant un milieu fluide dont le gradient thermique vertical entraîne une répartition de masse spécifique $\bar{p}(z)$, faiblement décroissante en fonction de la profondeur (voir fig. $1 \mathrm{c}$ ). Les mouvements turbulents dans la direction verticale sont encore influencés par la pesanteur, mais les effets sont de signe opposé à celui de ceux décrits en 1.2. Une fois que la particule s'est écartée de son niveau initial, la force d'Archimède tend à l'en éloigner encore davantage; le travail effectué est à l'avantage de l'énergie cinétique du mouvement turbulent dont l'amplitude est accrue. On dit alors que le gradient thermique est instable, et la diffusivité turbulente apparente est plus élevée que dans la situation de stabiiité neutre.

\section{5 - Situation de forte instabilité}

Lorsque le gradient thermique instable devient suffisamment élevé, et le gradient de vitesse suffisamment faible, les mouvements turbulents sont progressivement dominés par les forces dues à la pesanteur. L'écoulement tend alors vers un état de «forte instabilité 》, ou encore de « convection naturelle pure $\gg$ (free convection, en anglais).

1.5.1 - L'expérience montre alors que les structures turbulentes s'organisent, à partir de la surface où le flux de chaleur est appliqué, en un ensemble d'éléments convectifs (ou «panaches», «plumes» ou «thermals»), possédant des vitesses verticales appréciables vis-à-vis du fluide environnant et séparés par des zones plus étendues où s'opèrent les courants de retour nécessaires (voir le schéma de la fig. 3). Dans les océans, l'instabilité résulte presque toujours d'un refroidissement des couches superficielles sous l'effet des interactions océans-atmosphère. La convection libre se traduit alors par des «plongées » d'eau de surface, entraînant l'apparition d'une couche mélangée dont la limite inférieure correspond à une thermocline. Pour un refroidissement intense s'opérant sur une vaste étendue, le mouvement convectif atteint les couches océaniques les plus basses, constituant ainsi les véritables «sources d'eaux profondes» dont l'existence a été constatée en certains points du globe (cf. Lacombe, 1965).

1.5.2 - Le processus de convection naturelle s'amorce de lui-même, à partir de petites perturbations d'un milieu fluide initialement au repos, lorsque le nombre de Rayleigh de la région considérée, défini par:

$$
\mathrm{Ra}=-g \cdot \Delta \bar{\rho} \cdot \Delta \mathrm{z}^{3} / \bar{\rho} \lambda \nu
$$

franchit un certain nombre de valeurs critiques. L'instabilité initiale engendre des cellules convectives régulières (les "cellules de Bénard»), puis, à mesure que le nombre de Rayleigh augmente, les mouvements deviennent de plus en plus complexes, pour finalement atteindre le stade de la turbulence proprement dite (cf. Turner, 1973, ch. 7). 
1.5.3 - Les mouvements turbulents verticaux sont ici presque exclusivement d'origine gravitationnelle. La corrélation entre les fluctuations de la vitesse verticale $w^{\prime}$, et celles de la température $\theta^{\prime}$, qui sont la cause des fluctuations de densité responsables du mouvement, est donc nécessairement élevée. Par ailleurs, le gradient vertical de la vitesse moyenne étant limité, les fluctuations de la quantité de mouvement horizontale sont à la fois faibles et pratiquement découplées du mouvement vertical (voir Zilitinkevich, 1973). Ceci entraîne deux conséquences:

- Tout d'abord, la situation de convection libre correspond à un transfert turbulent vertical de chaleur très efficace. Il en résulte une homogénéisation rapide du champ des températures, dont un gradient ne pourra se maintenir que si un flux de chaleur suffisant est appliqué en permanence aux limites de la région considérée. On notera le contraste entre le déséquilibre inhérent à la situation instable, et l'état d'équilibre correspondant à la situation stable décrite en 1.3.1.

- Ensuite, les diffusivités verticales apparentes des contaminants scalaires contribuant aux variations de densité (comme la température ou la salinité) sont très supérieures à celle relative à la quantité de mouvement. Les nombres de Prandtl et de Schmidt effectifs sont donc ici faibles, à l'opposé de ce qui avait été dit en 1.3 .3 pour un état de stratification stable.

\section{2 - Les équations \\ gouvernant l'évolution quantitative des transferts turbulents}

\section{1 - Les particularifés de l'équation d'étał de l'eau de mer}

La masse spécifique de l'eau de mer dépend, non seulement de la température et de la salinité, mais encore de la pression et éventuellement de la concentration de certains produits ou matériaux contaminants (ce dernier point pourrait être important dans des problèmes de rejets polluants). Nous ne retenons ici que les deux premières variables, dont la figure 4 schématise l'influence.

Il est clair que les variations de densité, qui constituent le facteur important du point de vue des transferts turbulents, dépendent, non seulement des gradients thermiques, mais encore des gradients de salinité, et ceci dans des proportions qui dépendent fortement des valeurs moyennes de la température et de la salinité au point considéré. On peut donc conclure dès à présent que l'étude des effets des gradients thermiques ne peut être dissociée, en général, de celle des effets des gradients salins, et que les deux variables doivent être considérées ici simultanément.

\section{2 - La méthode générale suivie}

La description de l'évolution du milieu marin nécessite, dans le présent contexte, la connaissance, en fonction du temps et de l'espace, des variations de sept grandeurs fondamentales: masse spécifique $\rho$, pression $p$, température $\theta$,

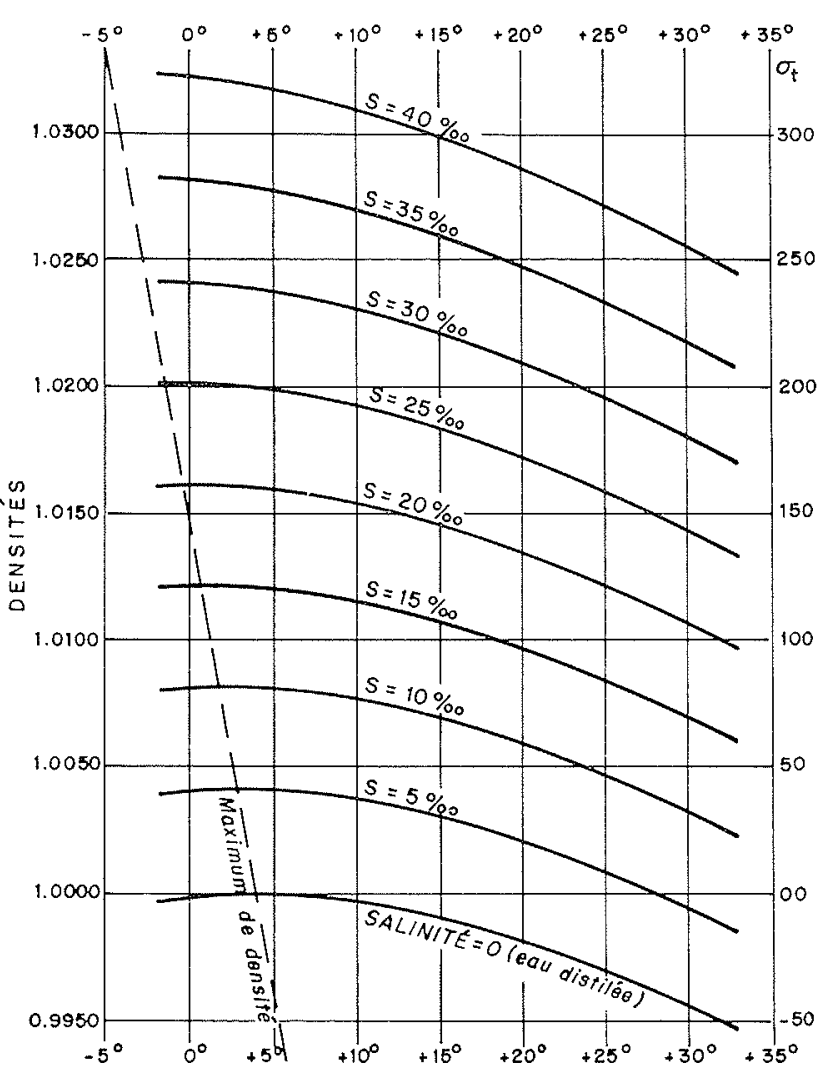

4/ L'équation d'état de l'eau de mer, $p=p(s, 0)$ Extrait de H. Lacombe : "Océanographie physique" (1965) Gauthier-Villars, Paris.

salinité $s$, et composantes de la vitesse $U_{i}$. Ces variations obéissent à un système de sept équations: d'état, de conservation de la masse, du mouvement (trois), de conservation du sel, et de conservation de l'énergie. Bien que déjà relativement simplité (cf. Eckart, 1962, Phillips, 1965), ce système est beaucoup trop compliqué pour que l'on en recherche directement des solutions.

Il doit donc être simplifié, et l'on utilise pour cela unc méthode connue sous le nom d'approximation de Boussinesq. Elle consiste à admettre que les quatre premières grandeurs fondamentales s'écartent peu des valeurs qu'elles posséderaient pour un "océan de référence», au repos et d'entropie et salinité constantes. On démontre (voir, par exemple, Dutton et Fitchl, 1969) que cette approximation est excellente si les caractéristiques du milieu de référence sont convenablement choisies : on peut admettre ici qu'elles correspondent à :

$$
\begin{gathered}
\rho=\rho_{0}=\text { Cte. } \quad, \quad \theta=\theta_{0}=\text { Cte. }, \quad s=s_{0}=\text { Cte. } \\
p=p_{0}(z)=p_{0}+\rho_{0} g z
\end{gathered}
$$

L'équation d'état très complexe schématisée par la figure 4 est alors représentée par l'approximation linéaire

$$
p=p_{0}\left\{1+\beta_{\theta_{0}}\left(s-s_{0}\right)+\beta_{\theta_{0}}\left(\theta-\theta_{0}\right)\right\}
$$

et $\rho$ peut s'éliminer du système, ainsi ramené à six équations simplifiées pour les six inconnues instantanées: $U_{i}, p, \theta, s$.

Chacune de ces inconnues est alors décomposée, suivant 
la méthode classique de Reynolds, en la somme d'une valeur moyenne et d'une fluctuation turbulente de valeur moyenne nulle. Ces nouvelles variables sont alors portées dans les équations précédentes, dont on prend ensuite des valeurs moyennes. On obtient ainsi (voir, par exemple, Phillips, 1966, Monin et Yaglom, 1971) un système de six équations pour les variables moyennes: $\bar{U}_{i}, \bar{p}$, $\bar{\theta}$, et $\bar{S}$. Par suite de la non-linéarité des termes d'advection figurant dans les équations du mouvement, et celles de conservation du sel et de l'énergie, ces nouvelles équations comportent en outre douze inconnues moyennes supplémentaires: $\overline{u_{i}^{\prime} u_{j}^{\prime}}, \overline{\theta^{\prime} u_{j}^{\prime}}, \overline{s^{\prime} u_{j}^{\prime}}$. Ces termes nouveaux représentent précisément, à un facteur $\rho_{0}$ ou $\rho_{0} C_{p}$ près, les valeurs des flux turbulents de quantité de mouvement, de chaleur, et de sel auxquels nous nous intéressons.

Le nouveau système est donc malheureusement indéterminé. On procède alors de la façon suivante : chacune des équations «moyennes» est soustraite terme à terme de l'équation «instantanés 》 correspondante; on obtient ainsi des équations «aux fluctuations»; ces équations sont multipliées par chacune des fluctuations $u_{j}^{\prime}, \theta^{\prime}, s^{\prime}$, et les expressions résultantes sont prises en valeurs moyennes; après quelques manipulations algébriques, on obtient finalement un système d'équations, dites « aux moments du second ordre», gouvernant l'évolution de toutes les combinaisons de la forme $\overline{x^{\prime} y^{\prime}}$. Cette méthode classique (cf. Monin et Yaglom, 1971) ne permet pas de «fermer » le système, mais procure de précieuses informations sur les mécanismes physiques affectant les transferts turbulents, et notamment ici sur le ròle des gradients verticaux de température et de salinité (voir Coantic, 1973).

\section{3 - Les équations résultantes dans une situation simple}

Pour faciliter la discussion, nous allons considérer unc situation très simplifiée, stationnaire et homogène horizontalement, où la force de Coriolis est négligeable, la vitesse moyenne parallèle à $O x$ et les transferts s'opérant dans la direction verticale $O z$ (cf. Stewart, 1959, Coantic, 1973), et l'effet du rayonnement supposé négligeable. Il vient alors:

$$
\begin{gathered}
\vec{U}=\bar{U}(z), \bar{V}=\bar{W}=0, \bar{p}=\bar{p}(z) \\
\bar{S}=\bar{S}(z), \overline{\bar{\theta}}=\bar{\theta}(z) \\
\tau(z)=-\mu \partial \bar{U} / \partial z+\rho_{0} \overline{u^{\prime} w^{\prime}}=\tau_{0}=\text { Cte. } \\
\sigma(z)=-\rho_{0} \mathcal{O} \partial \bar{S} / \partial z+\rho_{0} \overline{s^{\prime} w^{\prime}}=\sigma_{0}=\text { Cte. } \\
\zeta(z)=-\lambda \partial \bar{\theta} / \partial z+\rho_{0} C_{p} \overline{\theta^{\prime} \overline{w^{\prime}}}=\zeta_{0}=\text { Cte. }
\end{gathered}
$$

$$
\frac{d}{d t}\left(1 / 2 \rho_{0} \bar{u}^{\prime 2}\right)=0=-\rho_{0} \overline{u^{\prime} w^{\prime}} \frac{\partial \bar{U}}{\partial z}+\overline{p^{\prime} \frac{\partial u^{\prime}}{\partial x}}
$$

+ des termes de transfert et de dissipation;

$$
\frac{d}{d t}\left(1 / 2 \rho_{0} \bar{v}^{\prime 2}\right)=0=\overline{p^{\prime} \frac{\partial v^{\prime}}{\partial y}}
$$

+ des termes de transfert et de dissipation;

$$
\begin{aligned}
& \frac{d}{d t}\left(1 / 2 \rho_{0} \bar{w}^{\prime 2}\right)=0 \\
& =\rho_{0} g\left(\beta_{s_{0}} \overline{s^{\prime} w^{\prime}}+\beta_{\theta_{0}} \overline{\theta^{\prime} w^{\prime}}\right)+\overline{p^{\prime}} \frac{\partial w^{\prime}}{\partial z} \\
& + \text { des termes de transfert et de dissipation; } \\
& \frac{d}{d t}\left(1 / 2 \rho_{0}\left[\bar{u}^{\prime 2}+\bar{v}^{\prime 2}+\bar{w}^{\prime 2}\right]\right)=\frac{d}{d t} \bar{c}^{\prime}=0 \\
& =-\rho_{0} \overline{u^{\prime} w^{\prime}} \frac{\partial \vec{U}}{\partial z}+\rho_{0} g\left(\beta_{s_{0}} \overline{s^{\prime} w^{\prime}}+\beta_{\theta_{0}} \overline{\theta^{\prime} w^{\prime}}\right) \\
& \text { - - des termes de transfert } \\
& \text { - la dissipation de } \bar{c}^{\prime} \text { en chaleur; } \\
& \frac{d}{d t}\left(\overline{s^{\prime \prime}}\right)=0=-2 \overline{s^{\prime} w^{\prime}} \frac{\partial \bar{S}}{\partial z} \\
& \text { f- des termes de transfert } \\
& \text { - un terme de dissipation moléculaire; } \\
& \frac{d}{d t}\left(\bar{\theta}^{\prime 2}\right)=0=-2 \overline{\theta^{\prime} w^{\prime}} \frac{\partial \bar{\theta}}{\partial z}
\end{aligned}
$$

$$
\begin{aligned}
& \frac{d}{d t}\left(\rho_{0} \overline{u^{\prime} w^{\prime}}\right)=0 \\
& =-\rho \bar{w}^{\prime 2} \frac{\partial \bar{U}}{\partial z}+\rho_{0} g\left(\beta_{s_{0}} \overline{s^{\prime} u^{\prime}}+\beta_{\theta_{0}} \overline{\theta^{\prime} u^{\prime}}\right)
\end{aligned}
$$

+ - des termes de transfert, de pression et de viscosité;

$$
\begin{aligned}
& \frac{d}{d t}\left(\rho_{0} \overline{s^{\prime} w^{\prime}}\right)=0 \\
& =-\rho_{0} \overline{w^{\prime \prime 2}} \frac{\partial \bar{S}}{\partial z}+\rho_{0} g\left(\beta_{s_{0}} \bar{s}^{\prime 2}+\beta_{\theta_{0}} \overline{s^{\prime} \theta^{\prime}}\right) \\
& \quad+\text { des termes de transfert, de pression } \\
& \quad \text { et moléculaires; } \\
& \frac{d}{d t}\left(\rho_{0} C_{p} \overline{\left.\theta^{\prime} w^{\prime}\right)}=0=-\rho_{0} C_{p} \overline{w^{\prime 2}} \frac{\partial \bar{\theta}}{\partial z}\right. \\
& \quad+\rho_{0} C_{p} g\left(\beta_{s_{0}} \overline{s^{\prime} \theta^{\prime}}+\beta_{\theta_{0}} \bar{\theta}^{\prime 2}\right) \\
& \quad+\text { des termes de transfert, de pression } \\
& \quad \text { et moléculaires; } \\
& \frac{d}{d t}\left(1 / 2 \rho_{0} \bar{U}^{2}\right)=0=-\rho_{0} \overline{u^{\prime} w^{\prime}} \frac{\partial \bar{U}}{\partial z} \\
& \quad+\text { des termes de transfert } \\
& \quad \text { - un terme de dissipation en chaleur. }
\end{aligned}
$$

\section{3 - Interprétation physique et discussion de paramètres de similitude}

\section{1 - La signification physique des divers fermes des équations}

Les expressions (9) à (12) traduisent simplement les propriétés de la «couche à flux constants», qui résultent des hypothèses retenues en 2.3 (cf. Monin et Yaglom, 1971). 
Les termes figurant aux seconds membres des expressions (13) à (22) entrent dans cinq catégories :

- Termes contenant le produit d'un moment du second ordre par le gradient moyen de l'une des grandeurs transportées: il s'agit des taux auxquels l'interaction entre les mouvements turbulents et les gradients produit le moment figurant au prenier membre.

- Termes contenant en facteur l'accélération de la pesanteur : il s'agit des taux auxquels le moment du premier membre est directement produit ou détruit sous l'action des forces d'Archimède.

- Termes de pression: nous n'avons explicité ici que ceux figurant dans les équations (13) à (15); leur somme est nulle en raison de l'équation de continuité, et ils ne figurent donc pas dans (16); ils représentent un mécanisme de redistribution de l'énergie cinétique entre les trois composants de la vitesse, et donc de tendance vers l'isotropie (pour une discussion des divers termes de ce type, voir Monin et Yaglom, 1971, Tennekes et Lumley, 1973, Coantic, 1973).

- Termes de transfert, qui sont tous du type : $\partial / \partial z(\ldots)$, et traduisent donc des mécanismes de transport d'un niveau à l'autre de l'écoulement considéré.

- Enfin, termes moléculaires représentant les taux auxquels la diffusivité moléculaire des diverses grandeurs transportables transfère, modifie, ou encore dissipe les moments du second ordre figurant aux premiers membres (cf. références ci-dessus).

\section{2 - Le bilan de l'énergie cinétique de la turbulence, et le nombre de Richardson de flux}

Si l'on examine les équations (13) à (16) et (22), il est clair que le niveau de l'énergie cinétique des mouvements turbulents dépend au premier chef de deux facteurs:

- Le taux, - $\rho_{0} \overline{u^{\prime} w^{\prime}}(\partial \overline{\mathrm{U}} / \partial z)$, auquel elle est produite, dans la direction $O x$, aux dépens de l'énergie cinétique du mouvement moyen.

- Le taux, $\rho_{0} g\left(\beta_{s_{0}} \overline{s^{\prime} w^{\prime}}+\beta_{\theta_{0}} \overline{\theta^{\prime} w^{\prime}}\right)$, auquel elle est produite ou détruite, dans la direction $O z$, par le travail des forces d'Archimède, aux dépens ou à l'avantage de l'énergie potentielle du milieu.

Nous trouvons donc ici l'expression quantitative du mécanisme physique décrit au $\S 1$. Le sens et l'importance relative des effets de la stratification des densités sur la structure de la turbulence peuvent être caractérisés par le quotient de ces deux taux, qui constitue le nombre de Richardson de flux (le signe est choisi par convention comme positil dans le cas stable):

$$
\mathrm{Rf}=g\left(\beta_{s_{0}} \overline{s^{\prime} w^{\prime}}+\beta_{\theta_{0}} \theta^{\prime} w^{\prime}\right) / \overline{u^{\prime} w^{\prime}} \partial \bar{U} / \partial z
$$

\section{3 - Les phénomènes de double diffusivité}

Compte tenu de la définition (1) des coefficients de diffusivité turbulente apparente, et des équations (5), (8) et (23), on démontre aisément que:

$$
\begin{gathered}
\operatorname{Ri}=g \frac{\beta_{s_{0}}(\partial \bar{S} / \partial z)+\beta_{\theta_{0}}(\partial \overline{0} / \partial z)}{(\partial \bar{U} / \partial z)^{2}} \\
\operatorname{Rf}=g \frac{\left(\frac{K_{S . \tilde{z}}}{K_{I, z}}\right) \beta_{s_{0}} \frac{\partial \bar{S}}{\partial z}+\left(\frac{K_{\theta z}}{K_{I J, z}}\right) \beta_{\theta_{0}} \frac{\partial \bar{U}}{\partial z}}{(\partial \bar{U} / \partial z)^{2}}
\end{gathered}
$$

Si l'influence du gradient vertical de salinité peut être négligée, on retrouve l'expression classique:

$$
\mathrm{Ri}=\left(\mathrm{K}_{U, i} / K_{\theta z}\right) \mathrm{Rf}=\operatorname{Pr}_{t} \mathrm{Rf}
$$

Comme Richardson l'a prévu, la turbulence ne peut subsister lorsque $\mathrm{Rf}$ dépasse la valeur $\perp 1$ (la valeur limite est en fait plus faible si l'on tient compte de la dissipation). Mais nous avons vu en 1.3 .3 (et l'on pourrait démontrer ceci à partir de (19) et (21) que $\mathrm{Pr}_{\text {t }}$ tend vers des valeurs élevées pour les fortes stabilités. Le paradoxe apparent d'une turbulence subsistant dans l'océan pour des nombres de Richardson de gradient très élevés, est ainsi éclairci.

Par ailleurs, on peut montrer à partir de (20) et (21) quo le rapport $K_{S, \approx} / K_{\theta, z}$ peut prendre des valeurs très différentes de l'unité, en raison notamment de la très grande diflérence qui existe entre les diffusivités moléculaires de la chaleur et du sel dans l'océan. Si donc nous nous trouvons dans une situation où les gradients verticaux de température et de salinité exercent des effets opposés sur la densité, il est parfaitement possible que les signes de Rf et de Ri soient différents. Les phénomènes de «doublediffusivité », décrits par Turner (1973), montrent qu'il est en effet possible d'engendrer des mouvements convectifs gravitationnels dans un milieu dont le gradient moyen de densité est stable.

\section{4 - La théorie de Monin et Obukhov ef les développements récents}

Cet exposé resterait incomplet s'il n'y était pas au moins fait mention de la théorie de Monin et Obukhov, en raison du róle essentiel qu'elle a joué, et continue à remplir, dans la compréhension des effets de la stratification sur les transferts turbulents. Le point de départ de cette théorie est la constatation que les mécanismes turbulents au voisinage d'une surface, dans un gradient thermique vertical, ne peuvent dépendre, en outre de la distance $z$, à cette dernière, que de quatre grandeurs physiques fondamentales:

- la masse spécifique: $\rho_{0}$;

— le «paramètre gravitationnel $»: g \beta_{\theta_{0}} ;$

- la vitesse de frottement: $U^{*}=\left(\tau_{0} / \rho_{0}\right)^{1 / 2}$;

- le flux de chalcur réduit: $\zeta_{0} / p_{0} C_{j p}=\overline{\theta^{\prime} w^{\prime}}=U^{*} \theta^{*}$.

Le seul paramètre caractéristique possédant la dimension d'une longueur que l'on puisse construire à partir de ces quatre grandeurs est la longueur de Monin-Obukhov:

$$
L=\frac{U^{* 3}}{x g \beta_{\theta_{0}} \zeta_{0} / \rho_{0} C_{p}}=\frac{\rho_{0} C_{p} \theta_{0} U^{* 3}}{x g \zeta_{0}}
$$

L'analyse dimensionnelle prédit alors que toutes les variables caractéristiques des mécanismes de transfert turbulent doivent être, si elles ont été rendues sans dimensions en les multipliant par une combinaison convenable 
des grandeurs de référence, des fonctions universelles de la seule variable sans dimensions:

$$
\zeta=z / L
$$

Il vient, par exemple :

$$
\begin{aligned}
& \frac{\partial \bar{U}}{\partial z}=\frac{U^{*}}{x Z} \phi_{m}(z / L) \\
& \operatorname{Rf}=(z / L) / \phi_{m}(z / L)
\end{aligned}
$$

où $\phi_{m}$ est une fonction universelle, dont la forme a été étudiée expérimentalement et d'après diverses théories semi-empiriques. Pour une information plus complète sur les divers aspects de la théorie de Monin et Obukhov, et sur son application à l'océanographie, on pourra se reporter aux ouvrages classiques de Monin et Yaglom (1971) et de Phillips (1966). Notons en passant que l'une des difficultés dans la transposition de cette théorie au domaine océanique réside dans la définition du niveau de référence à partir duquel la variable fondamentale $z / L$ devra être comptée.

Mentionnons enfin le développement récent d'un certain nombre de méthodes de "modelage numérique » permettant d'opérer la fermeture du système complet des équations aux moments du premier et du second ordre (cf. Lumley et Khajeh-Nouri, 1974). L'emploi de ces méthodes, en conjugaison avec les données expérimentales actuelles, devrait permettre dans un avenir relativement proche de donner des solutions quantitatives correctes à certains problèmes de transfert turbulent dans un océan stratifié.

\section{Références}

CoAntic (M.). - Quelques commentaires sur les équations générales de la turbulence océanique (1973). Jorr. de Mécan., vol. 12, pp. 197-223.

Colloque IUCRM, Stockholm, 1969. - Spectra of meteorological variables. Radio-Science, vol. 4, n¹2, pp. 1099-1397.

Colloque IURCM, La Jolla, 1972. - Waves and turbulence instable layers and their eflects on EM propagation. Boundary-layer Meteorology, vol. 4, pp. 1-523, et vol. 5, pp. 1-256.

Dutron (J.A.) et Frrcil (G.H.). - Approximate equations of motion for gases and liquids (1969). Jour. Atmos. Sci., vol. 26, pp. 241-254.

ECKART (C.). - The equations of motion of sea-water (1962). Ii «the Sea», Hill M.N. Ed., Interscience, New York, pp. $31-41$.

LACombe (H.). - «Cours d'océanographiet physique» (1965) Gauthier-Villars, Paris.

Lumlex (J. L.) et KHAJeH-Nouri (B.). - Computational modeling of turbulent transport (1974). A paraître dans «Advances in Geophysics », vol. 18. Academic Press, New York.

Monyn (A.S.) et Yaglom (A. M.). - «Statistical fluid mechanics» (1971). M.I.T. Press, Cambridge.

Phillips (O. M.). - «The dynamics of the upper ocean» (1966). Cambridge University Press.

STEWART (R. W.). - The problem of diffusion in a stratified fluid (1959). Adv. in Geophysics, vol. 6, pp. 303-311.

Tennekes (H.) et Lumley (J. L.). - «A first course in turbulence» (1972). M.I.T. Press, Cambridge.

Turner (J.S.). - «Buoyancy effects in fluids》 (1973). Cambridge University Press.

ZiLitinkevich (S.S.). - Shear Convection. Boundary Layer Meteorology, vol. 3, pp. 416-423.

\section{Discussion}

président : M. J. DuporT

M. le Président remercie M. Conntic pour son très intéressant exposé et déclare ouverte la discussion.

M. le Professeur LACombe intervient en ces termes :

Le fameux problème de la «double diffusivité» m'a toujours laissé perplexe. Comment peuvent subsister, dans certaines régions marines, des couches intermédiaires chaudes et salées à toute époque de l'année, alors que, en présence de la «double diffusivité » une telle couche ne devrait pas être stable?

Or, de telles couches existent en permanence à 200 ou $400 \mathrm{~m}$ de profondeur dans la Méditerranée occidentale, et aussi dans l'Atlantique oriental en présence d'eaux méditerranéennes. Pourquoi ces couches persistent-olles indéfiniment?

D'après Turner, répond M. ConNTic, la double diffusivité peut, dans certaines circonstances, donner lieu à des phénomènes dits «de doigts salés», mais, dans d'autres circonstances, elle pent justement produire des «feuilletages de couches». Je ne suis pas assez familier avec la question pour vous répondre. Peut-être M. le Professeur Woods pourra-t-il nous informer sur ce sujet?

M. le Professeur Woods donne sori point de vue, en ces termes (traduction de son résumé en anglais) :

Je crois que les conditions, dans lesquelles la double diffusivité intervient, sont similaires à celles existant dans latmosphère dans un «anticyclone de blocage ou stationnaire» (blocking anticyclone), constitué par un tourbillon orageux persistant, en cours de subsidence, le fluide lourd étant caractérisé par des cisaillements faibles.

Si ma théorie de la turbulence géophysique est correcte, de tels tourbillons doivent persister dans l'océan durant de longues périodes - peut-être durant 1000 jours - Ainsi, les conditions favorables à la double diffusion peuvent vraisemblablement persister pendant une durée relativement longue, jusqu'à ce que - éventuellement - l'anticyclone stationnaire soit soumis à l'attaque extérieure d'un vigoureux cyclone dans lequel les cisaillements sont trop importants pour que le processus de double diftusivité puisse persister.

Ainsi, la question de savoir où la double diffusion prend naissance et où elle persiste peut trouver une réponse dans la considération de la turbulence géostrophique dans la région. Le temps nécessaire pour que la double diffusion détruise de tels grands tourbillons est plus long que celui mis par l'interaction des autres tourbillons géostrophiques pour produire le même résultat.

En raison des exigences de l'horaire, M. le Président se voit dans l'obligation de clore la discussion; il signale que les contributions écrites à celle-ci peuvent être adressées au Secrétariat de la S.H.F. qui en assurera la publication. Il donne ensuite la parole à $\mathrm{M}$. GoNELLA pour l'exposé de sa communication. 